\title{
ANÁLISE DA RELEVÂNCIA DA PROPRIEDADE SEMÂNTICA RECIPROCIDADE NOS VERBOS RECÍPROCOS INTRANSITIVOS DO PORTUGUÊS BRASILEIRO
}

\author{
The semantic property reciprocity in the intransitive reciprocal \\ verbs of Brazilian Portuguese
}

Thaís BECHIR ${ }^{1}$

Resumo: Os verbos recíprocos (VR) são tratados como uma classe na literatura por possuírem uma propriedade semântica - a reciprocidade - que licencia uma composição sintática: a alternância simples-descontínua. Nesta composição, os eventos denotados pelos verbos podem alternar entre as formas simples, em que os participantes da reciprocidade são denotados por apenas um argumento (João e Maria conversaram), e descontínua, em que os participantes são denotados por dois argumentos, sendo um deles preposicionado (João conversou com Maria). Objetivamos analisar os VR intransitivos e verificar se eles podem ser agrupados em uma só classe no português brasileiro. Intencionamos propor representações semânticas para as classes encontradas, valendo-nos da decomposição de predicados, além de apresentar uma discussão acerca do estatuto da propriedade semântica reciprocidade. Propusemos duas estruturas de decomposição de predicados para os VR intransitivos analisados, demonstrando que eles pertencem a duas classes distintas. Concluímos que os VR não formam uma classe e que a propriedade de reciprocidade, apesar de fazer parte do sentido idiossincrático de alguns verbos, é gramaticalmente irrelevante.

Palavras-chave: Semântica Lexical. Classes verbais. Reciprocidade. Verbos Recíprocos.
Abstract: The reciprocal verbs (RV) are treated as a verb class in the literature for having a semantic property - reciprocity - that allows a syntactic composition: simple-discontinuous alternation. In this composition, the events denoted by the verbs can switch between the simple form, in which the participants of the reciprocity are denoted by only one argument ('John and Mary talked'), and a discontinuous form, in which the participants are denoted by two arguments, and one of them is prepositioned ('John talked to Mary'). We aim to analyze the intransitive RVs and to verify if they can be grouped into a single class in Brazilian Portuguese. We intended to propose semantic representations for the classes we have found, by means of predicate decomposition metalanguage, in addition to presenting a discussion about the status of the semantic property reciprocity. We have proposed two predicate decomposition structures for the intransitive RVs analyzed, demonstrating that they belong to two distinct classes. We conclude that RVs do not form a class and that the property of reciprocity, despite being part of the idiosyncratic sense of some verbs, is grammatically irrelevant.

Keywords: Lexical Semantics. Verbal classes. Reciprocity. Reciprocal Verbs.

1 Bechir. UFMG. E-mail: thais.carvalhobechir@gmail.com. ORCID ID: https://orcid.org/0000-0003-08267044. 
- Análise da relevância da propriedade semântica reciprocidade nos verbos recíprocos intransitivos do Português Brasileiro

\section{Introdução}

Os verbos recíprocos (VR) são tratados como uma classe de verbos na literatura por possuírem, em seu sentido, a informação semântica de reciprocidade entre dois participantes (CHAFE, 1971; FILLMORE, 1972; ILARI, 1987; RAJAGOPALAN, 1987; CROFT, 1991; DOWTY, 1991; GLEITMAN et al., 1996), que licencia uma construção sintática: a "alternância simples-descontínua" (GODOY, 2008). Segundo Godoy (2008), a reciprocidade é uma propriedade semântica comum aos verbos que são passíveis de alternar sua estrutura sintática entre duas formas: a forma simples, em que os participantes da reciprocidade são denotados por um argumento plural (exemplos em (a) abaixo), e a forma descontínua, em que os participantes são denotados por dois argumentos, um em sua posição original, e um preposicionado, na posição de adjunção (exemplos em (b)). Por ser capaz de licenciar uma construção sintática, a alternância simples-descontínua, tal propriedade é considerada gramaticalmente relevante (GODOY, 2008). Exemplificamos os tipos de verbos recíprocos e a alternância simples-descontínua:

(1) Verbo recíproco transitivo, com reciprocidade no Al (argumento interno)

a. A cozinheira misturou os temperos. - forma simples

b. A cozinheira misturou o sal com a pimenta. - forma descontínua

(2) Verbo recíproco intransitivo, com reciprocidade no AE (argumento externo)

a. As vizinhas fofocaram. - forma simples

b. Uma vizinha fofocou com a outra. - forma descontínua

De acordo com Godoy (2008), para que um verbo seja classificado como recíproco, seu AE (no caso dos intransitivos) ou seu Al (no caso dos transitivos), na forma simples, deve sempre apontar um conjunto de participantes no mundo, em uma denotação plural, mesmo que o SN seja morfossintaticamente singular na sentença. A autora apresenta os seguintes exemplos (GODOY, 2008, p. 35; p. 83, grifo meu):

(3)
a. O casal flertou.
b. *João flertou.
c. Eles flertaram. 
(4) a. O pedreiro nivelou a superfície de pisos.

b. ${ }^{*} \mathrm{O}$ pedreiro nivelou o piso.

c. O pedreiro nivelou os pisos.

Em suma, para que um verbo seja classificado como recíproco, segundo Godoy (2008), ele deve:

a) possuir a propriedade semântica de reciprocidade entre dois participantes;

b) exigir um AE com denotação plural na forma simples, quando denotar reciprocidade ao seu $\mathrm{AE}$, ou exigir um $\mathrm{Al}$ plural na forma simples, quando denotar reciprocidade ao seu $\mathrm{Al}$;

c) ser passível de ocorrer na alternância simples-descontínua.

Entretanto, apesar dessas propriedades em comum, Cançado, Godoy e Amaral (2013) questionam o estatuto de classe verbal do grupo de VR. Adotando um critério de análise em que as classes devem ser uniformes tanto em comportamento semântico, como em comportamento sintático, as autoras apontam evidências de que os VR não apresentam tal uniformidade ou, pelo menos, não apresentam comportamento sintático exclusivo, compartilhando uma série de propriedades com verbos não recíprocos. Em uma análise exclusivamente dos VR transitivos, as autoras propõem que esses verbos fazem parte da classe de verbos de mudança de estado (CANÇADO; GODOY; AMARAL, 2013; CANÇADO, AMARAL, 2016), uma vez que possuem as propriedades semânticas e sintáticas desse grupo de verbos: acarretam uma sentença do tipo ficar estado, em que esse estado é morfologicamente relacionado ao verbo, e participam da alternância causativo-incoativa ${ }^{2}$ :

(5) Verbo de mudança de estado não recíproco
a. A menina sujou a roupa. - forma transitivo-causativa
b. A roupa ficou suja. - sentença do tipo ficar estado
c. A roupa (se) sujou. - forma intransitivo-incoativa 2 A alternância causativo-incoativa é um tipo de intransitivização em que o objeto direto passa para a posição
de sujeito, e o sujeito é apagado da estrutura. 
- Análise da relevância da propriedade semântica reciprocidade nos verbos recíprocos intransitivos do Português Brasileiro

(6) Verbo de mudança de estado recíproco
a. A cozinheira misturou os temperos. - forma transitivo-causativa
b. Os temperos ficaram misturados. - sentença do tipo ficar estado
c. Os temperos (se) misturaram. - forma intransitivo-incoativa

As autoras notam, ainda, que a alternância simples-descontínua, que caracterizaria esses verbos em termos sintáticos, pode também ocorrer com verbos não recíprocos, como o verbo roubar (CANÇADO; GODOY; AMARAL, 2013, p. 65):
a. O ladrão e a sua esposa roubaram a loja.
b. O ladrão roubou a loja com a sua esposa.

Assumindo a análise de Cançado, Godoy e Amaral (2013) para os VR transitivos e adotando a proposta de classificação verbal das autoras, é nossa intenção verificar se os VR intransitivos, assim como os VR transitivos já analisados, constituem uma classe verbal no PB. Na metodologia adotada para esta análise, consideramos relevantes apenas as propriedades semânticas capazes de licenciar construções sintáticas, seguindo os estudos da Interface Sintaxe-Semântica Lexical (ou apenas Semântica Lexical). Foram selecionados para esta pesquisa 31 VR intransitivos, retirados da pesquisa de Godoy (2008) ${ }^{3}$. A hipótese levantada é de que, assim como os VR transitivos, os VR intransitivos do PB, por si só, não formam uma classe verbal, sendo agrupados em classes maiores, comparáveis à dos verbos de mudança de estado.

Neste artigo, apresentaremos o arcabouço teórico que subjaz esta pesquisa; analisaremos os VR intransitivos por meio de testes sintático-semânticos, proporemos representações semânticas para as classes de VR intransitivos encontradas e discutiremos, por fim, a relevância gramatical da reciprocidade.

\footnotetext{
3 Tendo em vista que tomamos os dados coletados previamente por Godoy (2009) para a realização de nossa pesquisa, não objetivamos realizar, neste artigo, a validação de sua coleta. Ao contrário, partimos de sua coleta para discutirmos mais a fundo a propriedade semântica de reciprocidade e o comportamento dos verbos considerados recíprocos. Sendo assim, não temos como objetivo traçar uma discussão sobre transitividade ou sobre a controversa distinção entre complementos e adjuntos.
} 


\section{A classificação verbal}

Assume-se, na Semântica Lexical, que, na delimitação de uma classe, não basta observar o sentido específico dos verbos (FILLMORE, 1970; LEVIN, 1993; PESETSKY, 1995; LEVIN; RAPPAPORT HOVAV, 2005; GRIMSHAW, 2005; CANÇADO; AMARAL, 2016), mas é necessário observar se há propriedades semânticas presentes nos itens lexicais que são relevantes gramaticalmente, de maneira a impactar sua realização sintática.

Os verbos brigar e bater parecem ser semelhantes, a princípio, por denotarem algum tipo de movimento que ocorre entre pelo menos dois participantes: um dos participantes faz algum tipo de ação em relação a outro. Podemos pensar, então, que eles são suspeitos de possuírem as mesmas propriedades sintático-semânticas. No entanto, ao observamos o comportamento de ambos, vemos que apenas um deles ocorre na alternância simples-descontínua:

(8)

a. O meu irmão e o seu colega brigaram. - forma simples

b. O meu irmão brigou com o seu colega. - forma descontínua
a. * O meu irmão e o seu colega bateram. - forma simples
b. O meu irmão bateu no seu colega. - forma descontínua

Apenas o verbo brigar, em (8), aceita um argumento plural na posição de $A E$, na forma simples, podendo ser classificado como um verbo recíproco. Além disso, a partir de sua forma simples (8a), ele pode alternar para uma forma descontínua (8b). Já o verbo bater, em (9), não aceita um argumento plural em uma forma simples (9a), não podendo formar uma sentença que alterne entre as formas simples e descontínua. O verbo bater não pode, portanto, ser agrupado na mesma classe que o verbo brigar, uma vez que a propriedade semântica compartilhada por esses verbos não é gramaticalmente relevante.

Diferentemente, verbos que aparentemente não têm o mesmo conteúdo semântico, como quebrar e assustar, possuem as mesmas propriedades sintáticas, o que os agrupa em uma mesma classe:

(10) a. Os vândalos quebraram o busto do prefeito. - forma transitivo-causativa b. O busto do prefeito (se) quebrou. - forma intransitivo-incoativa 
- Análise da relevância da propriedade semântica reciprocidade nos verbos recíprocos intransitivos do Português Brasileiro

(11) a. Os vândalos assustaram o prefeito. - forma transitivo-causativa

b. O prefeito (se) assustou. - forma intransitivo-incoativa

Tanto em (10) como em (11), os verbos apresentam uma forma transitiva, que denota a causação de uma mudança de estado de um agente ou causa em um paciente (exemplos em (a)), e uma forma intransitiva, que denota apenas a mudança de estado do paciente (exemplos em (b)). Na verdade, as propriedades semânticas que agrupam verbos como quebrar e assustar são noções primitivas, como causa e mudança de estado. Tais verbos compartilham propriedades semânticas que são gramaticalmente relevantes, o que faz com que sejam agrupados em uma classe, a classe dos verbos de mudança de estado.

Cançado e Gonçalves (2016), seguindo Levin (2010), propõem que o nível de análise, o grain-size, também é relevante na classificação verbal. Levin (2010) já apontava que classes verbais podem ter diferentes tamanhos, dependendo do tipo e do nível do fenômeno linguístico levado em conta. As autoras assumem três níveis de análise para o agrupamento dos verbos, propostos a partir do nível de "granularidade" da classe verbal: coarse-grained, medium-graned e fine-grained. Cada um desses níveis se associa a determinados tipos de fenômenos linguísticos e sua escolha depende do tipo de análise que está sendo feita, mais ampla (coarse-grained), mediana (medium-grained) ou mais específica (fine-grained).

Segundo Cançado e Amaral (2016), a classificação do tipo coarse-grained agrupa os verbos por propriedades mais gerais, que podem estar presentes em verbos com diferentes representações semânticas. O conjunto de verbos transitivos é dado pelas autoras como um exemplo de classe que pode ser definida a partir desse nível de análise. Verbos transitivos diretos que apresentam um agente na posição de $\mathrm{AE}$, segundo Jackendoff (1972), podem ser passivizados ${ }^{4}$, o que os agrupa, de acordo com Cançado e Amaral (2016), em uma classe coarse-grained, visto que está sendo levada em conta apenas parte da estrutura desses verbos.

A análise do tipo medium-grained, por sua vez, considera toda a estrutura semântica de um grupo de verbos para que eles possam ser considerados uma classe verbal. Ela traz informações a respeito de classes consideradas mais canônicas na literatura. Um bom exemplo deste tipo de classificação é a classe dos verbos de mudança de estado, já exemplificada acima. Esses verbos possuem a propriedade sintática de participarem da alternância causativo-incoativa.

4 Isso não implica, contudo, que apenas verbos com agente na posição de sujeito podem ser passivizados. 
Por fim, a classificação do tipo fine-grained baseia-se apenas em sentidos idiossincráticos de alguns tipos de verbos que, de alguma maneira, licenciam determinadas construções sintáticas. O exemplo dado por Cançado e Amaral (2016) para elucidar essa classe são os VR. As autoras assumem que os VR, por si só, não formam uma classe do tipo coarse-grained ou medium-grained por não apresentarem nenhuma semelhança em sua estrutura argumental. Elas consideram, entretanto, que esses verbos são uma classe em uma análise do tipo fine-grained, já que, a partir da propriedade semântica de reciprocidade que eles abarcam, possuem a possibilidade de alternar entre as formas simples e descontínua. A propriedade semântica de reciprocidade não está presente na estrutura dos VR, mas é uma propriedade que faz parte de seu sentido idiossincrático.

Em suma, em um nível medium-grained, temos uma classe onde estão agrupados os VR transitivos, juntamente com outros verbos do PB: a classe dos verbos de mudança de estado. Ou seja, segundo Cançado, Godoy e Amaral (2013) os VR, por si só, não formam uma classe do tipo medium-grained, mas estão agrupados em uma classe deste tipo junto a outros verbos. No entanto, Cançado, Godoy e Amaral (2013) consideram que os VR transitivos, por si só, formam uma classe em um nível mais restrito, fine-grained, por possuírem uma propriedade semântica que tem impacto na sintaxe.

Baseando-nos nessas definições, objetivamos verificar se os VR intransitivos, não levados em conta na análise das autoras, podem ser considerados uma classe canônica, do tipo medium-grained, no PB.

\section{A linguagem de decomposição de predicados primitivos}

As informações sintáticas e semânticas de um verbo e de uma classe de verbos, ou seja, sua estrutura argumental, podem ser representadas a partir de diferentes metalinguagens. Alguns autores, começando por Fillmore (1968), propõem que a representação das estruturas argumentais dos verbos pode se dar a partir de uma lista de papéis temáticos. Em contrapartida, autores como Jackendoff (1983, 1990), Pinker (1989), Levin e Rappaport Hovav (2005), Cançado, Godoy e Amaral (2013), entre outros, adotam uma representação da estrutura argumental de um item verbal por meio da decomposição de seu significado em predicados primitivos, reforçando a ideia de que o sentido dos verbos é composicional. Os elementos da sintaxe dessa metalinguagem são os predicados, seus argumentos e seus modificadores e sua sintaxe é simples: um predicado pede um ou dois argumentos para saturar seu sentido e pode ser modificado. Segundo Cançado e Amaral (2016), a decomposição de predicados representa de maneira 
- Análise da relevância da propriedade semântica reciprocidade nos verbos recíprocos intransitivos do Português Brasileiro

mais complexa a semântica dos verbos. A partir dela, podemos observar a complexidade do evento denotado pelo verbo (se ele é ou não causativo) ${ }^{5}$, o papel temático de seus participantes e seu aspecto lexical.

Como vimos, os verbos de mudança de estado podem ser considerados uma classe por compartilharem a propriedade semântica de representarem um evento em que uma entidade muda de estado, acarretando o sentido ficar estado, e por serem causativos, contendo dois subeventos em seu sentido. Além disso, os verbos dessa classe podem alternar entre as formas transitiva e intransitiva, na alternância causativo-incoativa. Sua estrutura é dada abaixo (CANÇADO; GODOY; AMARAL, 2013, p. 93):

$$
\begin{aligned}
& \text { a. v: [[X ACT] CAUSE [BECOME }[\mathrm{Y}<S T A T E>]]]^{6} \\
& \text { b. amassar: [[X ACT] CAUSE [BECOME }[\mathrm{Y}<A M A S S A D O>]]]
\end{aligned}
$$

Na representação de (12b), podemos notar que o sentido do verbo amassar foi decomposto em elementos menores de sentido: os predicados primitivos (ACT, CAUSE e BECOME), as variáveis ( $\mathrm{X}$ e $\mathrm{Y}$ ) e a raiz "AMASSADO", que pertence à categoria ontológica dos estados, representada por STATE na estrutura da classe, em (12a). As variáveis $X$ e $Y$ são argumentos que saturam o sentido dos predicados. A raiz "AMASSADO" é a parte idiossincrática do sentido do verbo, ou seja, é ela que diferencia o significado dos verbos pertencentes à mesma classe, tais como, quebrar e assustar. O constituinte [X ACT] está associado ao desencadeamento da ação, enquanto o elemento [BECOME Y] denota o resultado da ação. O metapredicado CAUSE expressa a relação de causação entre a ação e seu resultado final. A raiz AMASSADO, por fim, denota o estado final do evento de amassar.

A estrutura dada em (12b), para o verbo amassar, pode ser representada pela seguinte sentença:

(13) A estudante amassou o papel.

5 Verbos causativos possuem uma relação causal necessária entre dois subeventos que compõem a semântica de um mesmo verbo: a ação e o resultado (CANÇADO; AMARAL, 2016). Em um verbo causativo como amassar, temos: [SUBEVENTO 1 a ação [SUBEVENTO 2 o resultado de algo ficar amassado]].

6 As estruturas de decomposição de predicados são apresentadas em inglês, língua em que foram propostas, para reforçar seu caráter universal, ou seja, não importa a língua em que aparecem, os predicados possuem o mesmo sentido (PINKER, 1989; CANÇADO; AMARAL, 2016). 
Em (13), temos que os argumentos do verbo amassar, a estudante e o papel estão associados às variáveis $\mathrm{X} \mathrm{e} \mathrm{Y,} \mathrm{respectivamente.} \mathrm{O}$ constituinte [X ACT] é representado pelo fato de a estudante agir e a parte da estrutura [BECOME $[\mathrm{Y}<\mathrm{AMASSADO}>]]$ é representada pelo fato de que, se a estudante amassou o papel, então o papel tornou-se amassado. $\mathrm{O}$ metapredicado CAUSE, por fim, representa o fato de que a estudante amassar o papel causa nele uma mudança de estado.

Adotaremos, neste trabalho, a decomposição de predicados como metodologia de análise da semântica dos VR intransitivos, propondo uma representação de seu sentido.

\section{Propriedades semânticas e sintáticas dos VR intransitivos}

Nesta seção, analisaremos os VR intransitivos ${ }^{7}$ a partir de testes sintáticosemânticos. Com o intuito de observar quais propriedades semânticas podem ter impacto na sintaxe de uma língua, pareceu-nos relevante a realização de análises aspectuais com relação aos VR intransitivos. A partir dessas análises, objetivamos observar a relação do aspecto lexical desses verbos com sua estruturação sintática.

O aspecto lexical, diferentemente do aspecto gramatical, é uma propriedade que vem especificada na entrada lexical dos itens verbais. Por ser inerente ao sentido do verbo, ele não vem marcado morfossintaticamente. Vendler (1967) propôs um sistema de aspecto lexical que é, ainda hoje, o mais aceito pelos linguistas. Segundo o autor, os verbos podem ser divididos em quatro classes aspectuais, a saber, estados, atividades, accomplishments e achievements. Essas quatro classes aspectuais se diferenciam em relação a três pares de valores aspectuais: estatividade $x$ dinamicidade; pontualidade $x$ duratividade; telicidade $x$ atelicidade. De acordo com a classificação de Vendler (1967) e seus valores aspectuais, os estados são estativos, durativos e atélicos. As atividades são dinâmicas, durativas e atélicas. Os accomplishments são dinâmicos, durativos e télicos. Os achievements são dinâmicos, pontuais e télicos.

Baseando-nos na proposta de Vendler (1967), analisamos os esquemas temporais definidos pelos VR intransitivos ${ }^{8}$. A partir da análise, percebemos que a diferença de valor aspectual entre eles tem relação com o par 'estatividade x dinamicidade'. Vejamos:

\footnotetext{
7 Os 31 VR intransitivos retirados de Godoy (2008) para serem analisados neste trabalho são: brigar, brindar, cochichar, coexistir, coincidir, combinar, comerciar, concordar, conflitar, contracenar, conversar, conviver, coocorrer, cooperar, corresponder, destoar, dialogar, discordar, discrepar, divergir, duelar, empatar, ficar, flertar, lutar, namorar, pactuar, prosear, rimar, tabelar e transar.
}

8 Assumimos, juntamente com Cançado e Amaral (2016), que os verbos podem ter uma leitura aspectual derivada em determinadas sentenças, decorrente da estruturação sintática. 
- Análise da relevância da propriedade semântica reciprocidade nos verbos recíprocos intransitivos do Português Brasileiro

(14) A natureza e o homem coexistem.

(15) Nossos horários de trabalho coincidem.

(16) As cores preto e vermelho combinam.

(17) O menino e sua irmã brigam muito.

(18) Leonardo DiCaprio e Kate Winslet contracenam juntos.

(19) Mike Tyson e Evander Holyfield lutam muito.

Em (14), (15) e (16), temos que os verbos coexistir, coincidir e combinar denotam situações que não necessitam de nenhuma força ou movimento para se manterem (são estativos), apresentam situações que se estendem em um período de tempo (são durativos) e não apresentam um resultado final (são atélicos). Nos verbos atélicos, cada instante é igual ao seu todo e não é preciso atingir um resultado final para que suas situações sejam verdadeiras: se dividirmos em três tempos as situações de coexistir, coincidir e combinar, teremos que $t_{1}=t_{2}=t_{3}$. De maneira semelhante, brigar, contracenar e lutar, em (17), (18) e (19), descrevem eventos que se estendem em um período de tempo (são durativos) e, também, não apresentam um resultado final (são atélicos). No entanto, podemos notar que esses verbos denotam situações que necessitam de uma força para continuarem a ocorrer (são dinâmicos), o que os diferencia dos verbos estativos coexistir, coincidir e combinar. Sendo assim, sem o impacto de alguma força ou movimento, brigar, contracenar e lutar não são eventos possíveis de se manterem no mundo. Feita esta análise, percebemos que coexistir, coincidir e combinar são verbos de estado, enquanto brigar, contracenar e lutar são verbos de atividade.

Concluímos, portanto, que os VR intransitivos do PB se diferenciam entre verbos com o aspecto lexical de atividade e verbos com aspecto lexical de estado. A distinção entre as características aspectuais desses verbos é um primeiro indício de que não se trata de uma única classe verbal.

Ainda, com a finalidade de compreender melhor o comportamento dos VR intransitivos de atividade, seguimos a proposta de Amaral e Cançado (2015). As autoras apontam evidências da diferença de comportamento entre dois tipos de verbos agentivos de atividade, que foram considerados por vários autores como pertencentes a uma mesma classe (RAPPAPORT HOVAV; LEVIN, 1998, 2010; PINKER, 1989; GRIMSHAW, 2005): verbos do tipo correr e verbos do tipo escrever. 
Uma das evidências dadas pelas autoras é a relação entre estes verbos e um objeto cognato: enquanto os verbos do tipo correr aceitam um objeto cognato, os verbos do tipo escrever não. Os objetos cognatos ou os objetos hipônimos, que também são aceitos pelos verbos do tipo correr, não são argumentos do verbo e, por isso, se comportam de forma diferente (JONES, 1988). Sendo assim, sua presença na posição de AI evidencia a existência de um nome contido dentro dos verbos, bem como a natureza agentiva de seu AE.

Objetivando observar a qual grupo de verbos agentivos de atividade os VR intransitivos fazem parte, testamos se eles aceitam a presença de um objeto cognato em sua estrutura sentencial. Como resultado, obtivemos que os VR intransitivos de atividade de nossa análise aceitam um objeto cognato ou hipônimo e são, portanto, verbos do tipo correr. Observemos alguns exemplos (em itálico, temos o objeto cognato):

(20) Os atletas correram a corrida de São Silvestre.

(21) O menino e sua irmã brigaram uma briga feia.

(22) Leonardo DiCaprio e Kate Winslet contracenaram uma cena emocionante.

(23) Mike Tyson e Evander Holyfield lutaram uma verdadeira luta.

Podemos evidenciar a diferença do comportamento semântico-sintático entre os VR intransitivos de atividade e os VR intransitivos de estado, quando notamos que os últimos não formam sentenças gramaticais quando acrescidos de um objeto cognato:

(25) * Nossos horários de trabalho coincidem uma coincidência certeira.

(26) * As cores preto e vermelho combinam uma combinação perfeita.

Além disso, a diferença de comportamento dos VR intransitivos de atividade em relação aos VR intransitivos de estado pode ser reafirmada a partir de um teste proposto por Jackendoff (1990). Segundo o autor, um argumento que se encaixa em uma estrutura do tipo "o que x fez foi..." pode ser identificado como um agente. Assim, para observar se os verbos de nossa análise eram agentivos, testamos se seus AE se encaixam neste tipo de estrutura: 
- Análise da relevância da propriedade semântica reciprocidade nos verbos recíprocos intransitivos do Português Brasileiro

(27) O que o menino fez foi brigar com sua irmã.

(28) O que o Leonardo DiCaprio fez foi contracenar com a Kate Winslet.

(29) O que o Mike Tyson fez foi lutar com o Evander Holyfield.

(30) *O que o homem fez foi coexistir com a natureza.

(31) * O que meu horário fez foi coincidir com o seu.

(32) * O que a cor preta fez foi combinar com a cor vermelha.

A partir do teste acima, notamos que os verbos de atividade, (27) a (29), são agentivos. Os verbos de estado, (30) a (32), ao contrário, não aceitam um agente na posição de $A E$, ou seja, não são agentivos. Este teste reforça a ideia de que os VR intransitivos de atividade fazem parte de um dos grupos de verbos de atividade agentivos de Amaral e Cançado (2015).

O objeto cognato também é utilizado, na literatura, para diferenciar os dois tipos de verbos intransitivos, segundo a 'Hipótese Inacusativa' (PERLMUTTER, 1978): os verbos inacusativos e os verbos inergativos. Podemos dizer que não se pode acrescentar aos verbos inacusativos um objeto cognato, uma vez que a função de objeto já está sendo exercida em sua estrutura. Os verbos inergativos, por sua vez, aceitam um cognato na posição de objeto direto, uma vez que eles não possuem essa função sintática já exercida em sua estrutura.

Sabendo-se que todo objeto direto tem a característica de ser construído no particípio, temos que apenas os verbos inacusativos aceitam essa construção. Os verbos inergativos, por possuírem um "sujeito verdadeiro", não podem ser construídos no particípio. Vejamos a diferença de comportamento entre um verbo recíproco de estado (33) e um verbo recíproco de atividade (34) abaixo:

(33) a. As cores branco e preto combinam.

b. *As cores preto e vermelho combinam uma combinação perfeita.

c. As cores combinadas.

(34) a. Mike Tyson e Evander Holyfield lutaram.

b. Mike Tyson e Evander Holyfield lutaram uma verdadeira luta.

c. * Os lutadores lutados. 
Acima, demonstramos que os verbos de estado como combinar não aceitam um objeto cognato (33b), mas podem ser construídos no particípio (33c), característica própria de verbos inacusativos. Já os verbos de atividade, como lutar, podem ser construídos com um objeto cognato (34b), mas não aceitam uma construção no particípio (34c), o que é comum a verbos inergativos. Considerando que os VR intransitivos de estado não aceitam um objeto cognato em sua estrutura, propomos que eles possuem a característica sintática de serem verbos inacusativos ${ }^{9}$. Já os VR intransitivos de atividade, por aceitarem um objeto cognato em sua estrutura, possuem a característica sintática de serem inergativos.

Em conclusão, apresentamos um quadro contendo os 31 VR intransitivos analisados e suas propriedades sintático-semânticas.

Quadro 1. Propriedades sintático-semânticas dos VR intransitivos

\begin{tabular}{|c|c|c|c|c|}
\hline VERBO & $\begin{array}{c}\text { ASPECTO } \\
\text { LEXICAL }\end{array}$ & $\begin{array}{c}\text { OBJETO } \\
\text { COGNATO }\end{array}$ & AGENTIVIDADE & INACUSATIVIDADE \\
\hline $\begin{array}{c}\text { brigar, brindar, } \\
\text { cochichar, } \\
\text { comerciar, } \\
\text { conflitar, } \\
\text { contracenar, } \\
\text { conversar, } \\
\text { dialogar, }\end{array}$ & atividade & & & \\
$\begin{array}{c}\text { discordar, duelar, } \\
\text { ficar, flertar, lutar, } \\
\text { namorar, pactuar, } \\
\text { prosear, tabelar, } \\
\text { transar. }\end{array}$ & & & agentivo & inergativo \\
\hline $\begin{array}{c}\text { coexistir, coincidir, } \\
\text { combinar, } \\
\text { concordar, conviver, } \\
\text { coocorrer, cooperar, } \\
\text { corresponder, } \\
\text { destoar, discrepar, } \\
\text { divergir, empatar, } \\
\text { rimar. }\end{array}$ & estado & 0 & não agentivo & inacusativo \\
\hline
\end{tabular}

Fonte: Elaboração própria

9 Apesar de não ser comum a classificação de verbos estativos em relação ao par inacusatividade $x$ inergatividade, nós apresentamos evidências de que os verbos estativos parecem se comportar como verbos inacusativos. É necessário, no entanto, estudos mais completos para que nossa proposta seja devidamente averiguada. Siloni (2001) também conclui, a partir de suas análises, que existem recíprocos inerentes que são predicados inacusativos, formados pela redução do papel temático externo. Não é nosso objetivo, entretanto, explicitar sua proposta neste artigo. 
- Análise da relevância da propriedade semântica reciprocidade nos verbos recíprocos intransitivos do Português Brasileiro

\section{A representação dos verbos recíprocos intransitivos}

Nesta seção, propomos a estrutura argumental dos VR intransitivos por meio da linguagem de decomposição em predicados primitivos. Em uma estrutura de decomposição de predicados, temos uma parte do significado que é recorrente entre os verbos de uma classe, e uma parte que representa o sentido idiossincrático dos verbos, que chamamos de "raiz" (RAPPAPORT HOVAV; LEVIN, 1998; LEVIN; RAPPAPORT HOVAV, 2005). As raízes podem ser argumentos, predicados ou modificadores doverbo. Além disso, elas pertencem a algumas categorias ontológicas, como STATE 'estado', EVENT 'evento', THING 'coisa', MANNER 'maneira' e PLACE 'lugar'. Essas categorias são representativas do sentido específico de uma classe de verbos e, assim como os predicados, possuem um número limitado (LEVIN; RAPPAPORT HOVAV, 2005).

Com o objetivo de descobrir a categoria ontológica dos VR intransitivos, realizamos o teste de retirar um nome de dentro dos verbos (CANÇADO; AMARAL, 2016). Cançado e Amaral (2016), seguindo as ideias iniciais de Jackendoff (1990), propõem que podemos extrair sintagmas correlatos ao sentido dos verbos, os quais evidenciam o componente semântico da raiz verbal. A partir deste teste, intencionamos observar a parte de sentido que os VR intransitivos carregam, reforçando a ideia de que o sentido de um item verbal é composicional. O teste feito para os VR de aspecto lexical de atividade está ilustrado abaixo:

(35) Brigar = briga.

(36) Contracenar $=$ cena.

(37) Lutar = luta.

Como chegamos à conclusão de que os VR de aspecto lexical de atividade fazem parte da mesma classe que os verbos do tipo correr, analisados por Amaral e Cançado (2015), entendemos que eles teriam a mesma estrutura e, portanto, a mesma categoria ontológica desses, sendo representados pela categoria ontológica EVENT 'evento'.

Abaixo, demonstramos o teste realizado para os VR de aspecto lexical de estado:

(38) Coexistir $=$ coexistência

(39) Coincidir $=$ coincidência.

(40) Combinar = combinação. 
Cançado (2012), baseando-se em Van Valin (2005), propõe para os verbos psicológicos do tipotemer a categoria ontológica THING "coisa". Pinker(1989) dá a estrutura do verbo estativo have "ter", considerado também um predicado primitivo na teoria, utilizando-se, de forma semelhante, da categoria ontológica THING para representar seu sentido. Seguindo a ideia desses autores que, apesar de possuírem propostas com enfoques distintos, tomam como ferramenta de base o decomposicionismo de Jackendoff ${ }^{10}$, levamos em conta a possibilidade de a categoria ontológica $\langle$ THING $>$ ser a representativa do sentido idiossincrático da classe dos VR intransitivos de estado.

Tendo realizado o teste do nome dentro dos verbos, realizamos um novo teste, com o intuito de descobrir o predicado primitivo adequado para estruturar os VR intransitivos de atividade e de estado. Por meio deste teste, objetivamos encontrar uma paráfrase do sentido dos verbos, fazendo uso dos nomes dos verbos que encontramos a partir do teste anterior (PINKER, 1989). Exemplificamos o teste de paráfrase ${ }^{11}$ para os VR intransitivos de atividade:

(41) Brigar = fazer uma briga.

(42) Contracenar = fazer uma cena.

(43) Lutar = fazer uma luta.

Vejamos agora o resultado das paráfrases para os VR intransitivos de estado:

(44) Coexistir $=$ ter coexistência.

(45) Coincidir = ter coincidência.

(46) Combinar = ter combinação.

A partir do teste do nome dentro dos verbos e do teste da paráfrase dos verbos, pudemos observar o comportamento dos VR intransitivos. Parece-nos possível, agora, discutir mais a fundo quais seriam suas categorias ontológicas e propor a eles um predicado primitivo.

\footnotetext{
10 Enquanto Pinker (1989) visa à construção de uma teoria de aquisição em alternâncias verbais, bootstrapping semântico e mudança linguística utilizando-se da decomposição, Cançado (2012) propõe uma análise dos verbos psicológicos a partir do decomposicionismo.

11 É importante observar que as paráfrases dos verbos podem variar. Reconhecendo-se essa possibilidade de variação, as paráfrases foram elaboradas levando em conta o aspecto lexical básico do verbo.
} 
- Análise da relevância da propriedade semântica reciprocidade nos verbos recíprocos intransitivos do Português Brasileiro

Os VR intransitivos de atividade são semântica e sintaticamente semelhantes aos verbos da classe de correr $^{12}$, cujo comportamento foi investigado por Amaral e Cançado (2015). Assim, a partir da aceitabilidade dos VR intransitivos de atividade quanto ao objeto cognato, concluímos que eles são estritamente agentivos e que eles têm a característica sintática de serem inergativos, pertencendo à mesma classe que os verbos do tipo correr. Amaral e Cançado (2015) propõem uma separação dos verbos agentivos de atividade em duas classes: os verbos do tipo correr seriam representados pela estrutura argumental [X DO $<E V E N T>$ ], enquanto os verbos do tipo escrever seriam representados pela estrutura $\left[\mathrm{X} \mathrm{ACT}_{\angle M A N N R>}\right]$. As autoras seguem a ideia de Harley (2005), mostrando que a classe dos verbos do tipo correr não pode conter MANNER 'maneira' como categoria ontológica. A evidência dada por elas para justificar a separação dos verbos intransitivos agentivos de atividade em duas classes distintas é o fato de que uma estrutura como [X ACT ${ }_{\text {SMANNER }}$ ] só pode derivar atividades durativas e verbos como pular, por exemplo, são atividades agentivas intransitivas pontuais (conhecidas como semelfactivos) ${ }^{13}$. Propõe-se que os verbos do tipo correr possuem, portanto, a raiz EVENT (ROSS, 1972; HARLEY, 2005). As autoras também demonstram que os verbos do tipo correr não podem conter o predicado primitivo ACT (RAPPAPORT HOVAV; LEVIN, 1998), por ele ser monoargumental, não deixando espaço na estrutura para um argumento-raiz do tipo EVENT. Propõe-se para eles, então, o predicado primitivo DO, já que ele é biargumental, requerendo uma variável e a raiz EVENT como argumentos (ROSS, 1972).

Visto que consideramos que os VR intransitivos de atividade pertencem à mesma classe dos verbos do tipo correr, propomos a eles a seguinte estrutura argumental (47a) e apresentamos alguns exemplos de verbos pertencentes a essa classe (47b, 47c, 47d):
a. $v:[\mathrm{X} \mathrm{DO}<E V E N T>]$.
b. brigar: $[\mathrm{X} \mathrm{DO}<B R I G A>]$.
c. contracenar: $[\mathrm{X}$ DO $<C E N A>]$.
d. lutar: $[\mathrm{X}$ DO $<$ LUTA $>]$.

Dada a estrutura de decomposição de predicados para os VR intransitivos de atividade, podemos agora discutir a estrutura dos VR intransitivos de estado, para os

12 São exemplos de verbos do tipo correr, os verbos dançar, chorar, pular e andar.

13 Assumiremos, juntamente com Levin (1999), Harley (2005) e Cançado e Amaral (2016), que os verbos semelfactivos como pular não formam uma quinta classe aspectual, mas são atividades que carregam o sentido idiossincrático da iteratividade em sua entrada lexical. 
quais já foram apresentados os testes de nome contido nos verbos (35-37) e paráfrase dos verbos (44-46). Na paráfrase dos verbos de estado, pudemos perceber uma regularidade com relação ao uso do verbo ter. Consideramos, então, a possibilidade de utilizarmos, para representar o sentido recorrente desta classe de verbos, o metapredicado HAVE, já utilizado por Pinker (1989) e Cançado (2012) em suas representações. Para os verbos psicológicos do tipo temer, Cançado (2012, p. 15) propõe o metapredicado TER e a categoria ontológica THING:

$$
\begin{aligned}
& \text { a. } v \text { : }[\mathrm{X} \text { TER }<\text { THING }>\text { por } \mathrm{Y}] \\
& \text { b. temer: }[\mathrm{X} \text { TER }<\text { TEMOR }>\text { por } \mathrm{Y}]
\end{aligned}
$$

Pinker(1989) propõe a existência do metapredicado HAVE, além do metapredicado $\mathrm{BE}$, já que, para ele, a propriedade de posse pode ser conceptualizada por meio desses dois predicados semânticos. Nesse sentido, o autor sugere que, cognitivamente, o estado HAVE é simplesmente o inverso do estado BE, tratando a location $^{14}$, ao invés do locatum $^{15}$, como seu "sujeito lógico" (PINKER, 1989). Abaixo, representamos a ideia do autor:

(49) BE: $\{$ posse/locatum, possuidor/location $\}$

(50) HAVE: \{possuidor/location, posse/locatum\}

Dadas as representações acima, notamos que o predicado BE deve ser utilizado em uma estrutura em que o AE indica posse e o Al indica o possuidor. Inversamente, a conceptualização da ideia de posse a partir do predicado HAVE deve ser feita quando temos um AE designando possuidor e um AI designando uma posse.

Pinker (1989) aponta outras diferenças entre os predicados BE e HAVE. Segundo o autor, enquanto o predicado BE pode ser definido normalmente por meio de um campo locacional, podendo ser estendido para um campo de posse, o predicado HAVE é normalmente definido a partir do campo de posse, podendo ser estendido para um campo locacional. A partir desta ideia, podemos notar que o predicado HAVE, definido a partir de um campo de posse, melhor define os VR intransitivos de estado:

(51) X ter coincidência: \{possuidor/location, posse/locatum\}

14 O termo location faz referência ao lugar, ou seja, nesse caso, ao possuidor.

$15 \mathrm{O}$ termo locatum faz referência à posse. 
- Análise da relevância da propriedade semântica reciprocidade nos verbos recíprocos intransitivos do Português Brasileiro

Em (51), o AE de ter, X, é o possuidor, enquanto seu Al, coincidência, é a posse. Isso justifica a nossa opção pelo metapredicado HAVE em contraposição à possibilidade de utilizarmos o metapredicado BE.

Considerando-se a vantagem do uso do predicado HAVE e com base nas estruturas dadas pelos autores apontados, propomos a estrutura de decomposição de predicados para os VR intransitivos de estado (52a) e apresentamos alguns exemplos de verbos pertencentes a essa classe (52b, 52c, 52d):
a. $v:[\mathrm{X} H A \bigvee E<T H I N G>]$
b. coexistir: [X HAVE $<$ COEXISTÊNCIA>]
c. coincidir: [X HAVE $<$ COINCIDÊENCIA $>$ ]
d. combinar: [X HAVE <COMBINAÇÃO $>$ ]

Encontradas as estruturas de decomposição de predicados para os VR intransitivos do PB, retomamos o nosso objetivo de discutir se esses verbos podem ser agrupados em uma classe verbal única, do tipo medium-grained. Podemos afirmar que os VR intransitivos, assim como os VR transitivos analisados por Cançado, Godoy e Amaral (2013), não formam uma única classe verbal medium-grained no PB, uma vez que eles possuem duas estruturas semânticas distintas: [X DO $<E V E N T>$ ] e [X HAVE $<$ THING $>$ ]. Sendo assim, como vimos a partir dos testes executados, eles não compartilham as mesmas propriedades sintáticas e semânticas.

Destacamos que a classe de verbos com a estrutura [X DO <EVENT>] não é composta apenas de VR intransitivos de atividade, mas por outros verbos intransitivos agentivos de atividade, analisados por Amaral e Cançado (2015). Assim, os VR intransitivos de atividade, por si só, não formam uma classe medium-grained, mas estão agrupados na classe [X DO <EVENT>] juntamente com outros tipos de verbos: os verbos do tipo correr, de Amaral e Cançado (2015). Além disso, há fortes indícios de que a classe de verbos formada a partir da estrutura [X HAVE $\langle$ THING $>$ ] também não é composta apenas de VR intransitivos estativos, visto que encontramos exemplos de verbos estativos intransitivos não recíprocos que parecem teresta mesma estrutura semântica: proceder =ter procedência ([X HAVE $<$ PROCEDÊNCIA>]); existir = ter existência ([X HAVE $<$ EXISTÊNCIA $>$ ]). As evidências apresentadas a partir desses exemplos apontam para o fato de que os VR intransitivos estativos, por si só, não formam uma classe medium-grained, mas estão agrupados na classe de estrutura [X HAVE $\langle$ THING $>$ ] juntamente com outros tipos de verbos estativos do PB. Uma análise mais fina dos demais verbos pertencentes a essa classe, no entanto, deve ser realizada em trabalhos futuros. 
Confirmamos, portanto, a hipótese levantada de que os VR intransitivos do PB não formam uma classe verbal, mas fazem parte de classes verbais mais amplas em um nível medium-grained, em concordância com a proposta de Cançado, Godoy e Amaral (2013). Na seção seguinte, discutimos a classificação dos VR intransitivos em um nível fine-grained.

\section{Os verbos recíprocos transitivos e intransitivos: uma classe verbal fine-grained?}

Cançado, Godoy e Amaral (2013) apresentaram evidências de que os VR possuem diferentes comportamentos sintático-semânticos:verbos do tipo conversarsão intransitivos e não acarretam um estado final, enquanto os verbos do tipo misturar acarretam ficar estado, participam da alternância causativo-incoativa e são bieventivos. A partir disso, as autoras concluíram que os VR não formam uma classe verbal no PB. Cançado e Amaral (2016), baseando-se nessa análise anterior, propõem que os VR não formam uma classe verbal canônica, do tipo medium-grained, no PB, mas uma classe de análise mais restrita, do tipo fine-grained, visto que os verbos desta classe possuem a propriedade semântica de reciprocidade, que licencia uma construção sintática: a alternância simples-descontínua.

Nesta seção, apresentaremos evidências de que o agrupamento dos VR em uma única classe verbal não é simples de ser fundamentado, nem mesmo em um nível mais restrito de análise. Uma primeira evidência vem do fato de que a própria caracterização do que seja um verbo lexicalmente recíproco é problemática. Segundo Godoy (2008), um verbo lexicalmente recíproco pode ser identificado a partir da relação de acarretamento entre as formas simples e descontínua, ou seja, se o verbo é recíproco, sua forma simples acarreta a descontínua. Porém, esse teste não se mostra suficiente para separar os verbos lexicalmente recíprocos daqueles cuja reciprocidade é sintática. Observemos os exemplos abaixo:
a. A mãe e o bebê dormiram. - forma simples
b. A mãe dormiu com o bebê. - forma descontínua
a. Mike Tyson e Evander Holyfield lutaram. - forma simples
b. Mike Tyson lutou com Evander Holyfield. - forma descontínua

De acordo com a análise de Godoy (2008), apenas o verbo lutar, em (54), é um verbo lexicalmente recíproco. O verbo dormir, em (53), seria considerado não recíproco em uma construção sintática recíproca. No entanto, podemos notar que os verbos dormir e lutar apresentam o mesmo comportamento com relação ao acarretamento: ambos, em sua forma simples, não acarretam sentenças descontínuas: 
- Análise da relevância da propriedade semântica reciprocidade nos verbos recíprocos intransitivos do Português Brasileiro

(55) A mãe e o bebê dormiram. トA mãe dormiu com o bebêt ${ }^{16}$.

(56) Mike Tyson e Evander Holyfield lutaram. -Mike Tyson lutou com Evander Holyfield.

Além disso, Godoy (2008) argumenta que os verbos lexicalmente recíprocos requerem, de maneira obrigatória, um argumento plural na posição de AE ou de Al em sua forma simples, como já apontamos. Entretanto, essa argumentação não se mostra válida para alguns dos verbos classificados como recíprocos pela autora:

(57) Galvão Bueno comentou a partida de futebol.

(58) Cristina discutiu os resultados de sua dissertação.

(59) Jorge joga futebol todas as quartas.

Nas sentenças acima, temos verbos que denotam reciprocidade ao seu AE. Podemos notar, a partir delas, que nem todos os verbos classificados por Godoy (2008) como lexicalmente recíprocos exigem que seu AE tenha uma denotação plural. Reforçando essa ideia, Siloni (2001) reconhece a existência de uma operação derivacional que reduz o $\mathrm{Al}$ e que forma VR nas diferentes línguas. O autor argumenta que esta operação se aplica em certas línguas na sintaxe e em outras no léxico, afirmando que os VR que não aceitam um argumento singular são os verbos sintaticamente recíprocos, e não os que possuem reciprocidade lexical.

As análises do teste do acarretamento e do argumento plural, juntamente com a proposta de Siloni (2001), enfraquecem a ideia de que os VR formam uma classe de verbos e mostram que a própria noção de reciprocidade lexical é difícil de ser definida, uma vez que os testes propostos na literatura para a identificação de tal propriedade não parecem se sustentar.

Cançado et al. (2013) mostram, além disso, como já apontamos, que a alternância simples-descontínua não é uma composição sintática comum apenas aos VR, podendo ocorrer com vários tipos de verbos no $\mathrm{PB}$. As autoras citam o verbo transitivo roubar (CANÇADO; GODOY; AMARAL, 2013, p. 65) e podemos citar, também, o verbo intransitivo jantar:

16 O símbolo F indica a ausência de acarretamento (CANN, 1993). 
a. João e Maria roubaram a loja. - forma simples

b. João roubou a loja com Maria. - forma descontínua

(61)
a. Cristina e Jorge jantaram. - forma simples
b. Cristina jantou com Jorge. - forma descontínua

Os verbos roubar e jantar não exigem um AE plural e não possuem a propriedade semântica de reciprocidade em seu sentido, no entanto, ocorrem nas formas simples e descontínua.

Ignácio (2009) trata a respeito do comitativo, um caso semântico pouco estudado, defendendo a ideia de que ele é um complemento do verbo. Segundo o autor, o comitativo é um termo nuclear que se caracteriza por participar da estrutura oracional na posição de complemento, porém com função idêntica à do AE da oração. O comitativo pode ser introduzido em uma sentença por meio da preposição com ou por meio da expressão junto com. Vejamos alguns exemplos de argumentos comitativos (marcados em itálico) (IGNÁCIO, 2009, p. 17, grifo nosso):

O patrão discutia com o empregado.

(63) O filho sofriajunto com a mãe a perda do pai.

(64) A Maria sempre saía com o João.

Seguindo a proposta do autor, em (62), tanto o AE quanto o comitativo são agentes; em (63), tanto o AE quanto o comitativo são pacientes e, em (64), o AE e o comitativo são temas.

Hurst (2010) trata do comitativo ao fazer uma análise das construções recíprocas do Swahili17. Segundo o autor, o comitativo possui propriedades tanto de argumento quanto de adjunto. Sendo assim, ele utiliza da concepção de a-adjunct 'a-adjunto', de Grimshaw (1990), para demonstrar que o comitativo possui esse status intermediário. O autor desenvolve sua análise concluindo que alguns predicados podem selecionar um comitativo como um argumento, ao invés de um a-adjunto. Ainda, o autor conclui que, para que o comitativo a-adjunto seja reanalisado como um argumento, o predicado deve requerer a participação de duas entidades que devem se engajar em uma relação

17 O suaíli ou suaíle, também chamado de suahíli e conhecido pelas formas vernáculas Swahili ou Kiswahili, é a língua bantu com o maior de número de falantes. 
- Análise da relevância da propriedade semântica reciprocidade nos verbos recíprocos intransitivos do Português Brasileiro

que seja compatível com a semântica comitativa ${ }^{18}$. Siloni (2012), por sua vez, não chega a mencionar o comitativo em seu estudo, mas afirma que a construção descontínua nem sempre é recíproca, argumentando que toda construção descontínua tem uma interpretação recíproca (simétrica), apesar de que ela pode, adicionalmente, ter uma leitura não recíproca. Segundo Dowty (2001), em uma alternância de diátese, há uma forma mais básica e geral que outra, semântica e sintaticamente. $\mathrm{O}$ autor exemplifica a forma marcada como X, em oposição à forma não marcada Y. Dowty (2001) já mostrava que muitos verbos ocorrem em $X$ e não ocorrem em Y. Em contrapartida, há alguns ou nenhum verbo que ocorre em $Y$, mas não ocorre em $X$.

Segundo Cançado, Godoy e Amaral (2013, p. 65), todos os VR "são participantes por excelência de composições sintáticas comitativas", mas podemos notar que nem toda composição sintática comitativa é feita a partir de um verbo lexicalmente recíproco. Abaixo, em (65), apresentamos um exemplo de um verbo lexicalmente recíproco que, por conseguinte, participa de uma construção sintática com o argumento comitativo em sua forma descontínua. Já em (66) e (67), temos exemplos de verbos que não são lexicalmente recíprocos, mas que, a partir de uma construção sintática com o argumento comitativo, em uma forma semelhante à forma descontínua, abarcam a propriedade de reciprocidade:

a. O patrão e o empregado discutiam. - forma simples

b. O patrão discutia com o empregado. - forma descontínua
a. O filho e a mãe sofriam a perda do pai. - forma simples

b. O filho sofria a perda do pai (junto) com a mãe. - forma descontínua

a. A Maria e o João sempre saíam. - forma simples

b. A Maria sempre saía (junto) com o João. - forma descontínua

Nos exemplos dados acima, podemos observar que tanto o verbo lexicalmente recíproco e, portanto, participante de uma composição comitativa, como em (65), quanto os verbos que possuem uma reciprocidade sintática e que participam da composição comitativa, como em (66) e (67), podem alternar entre as formas simples e descontínua. Nesse sentido, se é a alternância simples-descontínua que agrupa os VR em uma mesma

$18 \mathrm{O}$ autor pontua que os verbos naturalmente simétricos e os verbos simétricos que são formados no léxico cumprem estes critérios, acrescentando que um verbo simétrico é aquele que requer prototipicamente dois participantes com participação semelhante no evento. 
classe (GODOY, 2008; CANÇADO; GODOY; AMARAL, 2013) e foram apresentados exemplos em que essa alternância ocorre também com verbos não recíprocos em uma construção comitativa, é questionável que os VR formem uma classe até mesmo em um nível mais restrito, do tipo fine-grained.

Concluímos que os VR não formam uma classe verbal no PB e que a reciprocidade, apesar de fazer parte do sentido idiossincrático de alguns verbos, não é gramaticalmente relevante. Ademais, discordamos de Cançado, Godoy e Amaral (2013) em relação à análise de que os verbos recíprocos sejam uma classe do tipo fine-grained, já que não há testes suficientes para o diagnóstico dessa propriedade semântica nos VR e considerando que a propriedade sintática que os agrupa, a alternância simples-descontínua, não é exclusiva a eles. As conclusões deste trabalho, portanto, vão além das conclusões de Cançado, Godoy e Amaral (2013): enquanto as autoras consideram que a reciprocidade é relevante gramaticalmente, agrupando verbos em uma classe fine-grained, nós consideramos que essa propriedade é irrelevante sintaticamente, não sendo capaz de agrupar verbos em classes em nenhum nível de análise.

\section{Considerações finais}

Observamos que os VR intransitivos se subdividem entre verbos de aspecto lexical de atividade e verbos de aspecto lexical de estado, sendo que apenas os primeiros aceitam um objeto cognato em sua estrutura. Além disso, fomos capazes de reconhecer que os VR intransitivos de atividade são inergativos e estritamente agentivos, enquanto os VR intransitivos de estado são inacusativos e não agentivos.

A partir do reconhecimento de que os VR intransitivos de atividade e os VR intransitivos de estado possuem um comportamento sintático-semântico distinto, diferentes representações semânticas foram propostas a eles. Para os VR intransitivos de atividade, propusemos a estrutura ' $v$ : [X DO $<E V E N T>$ ', considerando que eles fazem parte da mesma classe que os verbos do tipo correr, analisados por Amaral e Cançado (2015). Para os VR intransitivos de estado, por sua vez, foi proposta a estrutura ' $v$ : [X HAVE $<$ THING $>$ ]', com base em Pinker (1989) e em Cançado (2012).

Demonstramos que os VR não formam uma classe verbal no PB em nenhum nível de análise e que a reciprocidade, apesar de fazer parte do sentido idiossincrático de alguns verbos, não é uma propriedade gramaticalmente relevante, discordando de Cançado, Godoy e Amaral (2013). Os argumentos levantados para defender essa ideia são apresentados a seguir: 
- Análise da relevância da propriedade semântica reciprocidade nos verbos recíprocos intransitivos do Português Brasileiro

a) os VR transitivos, analisados por Cançado, Godoy e Amaral (2013), e os VR intransitivos analisados por nós, neste artigo, possuem um comportamento sintáticosemântico distinto entre si;

b) o teste do acarretamento, proposto por Godoy (2008), para separar os verbos lexicalmente recíprocos daqueles cuja reciprocidade é sintática, se mostrou ineficiente;

c) a argumentação de que os verbos lexicalmente recíprocos possuem a exigência sintática de um argumento plural na posição de $A E$ (quando denotam reciprocidade ao seu $\mathrm{AE}$ ) ou na posição de $\mathrm{Al}$ (quando denotam reciprocidade ao seu $\mathrm{Al}$ ) não se mostrou válida para parte dos VR analisados por Godoy (2008);

d) a alternância simples-descontínua, composição sintática que agrupa os verbos lexicalmente recíprocos em uma mesma classe, pode ocorrer com outros tipos de verbos, em uma composição sintática chamada de comitativa.

Por fim, nossa hipótese de que os VR intransitivos do PB não formam, por si só, uma classe verbal, foi corroborada, na medida em que eles foram agrupados em classes maiores: na classe dos verbos do tipo correr, analisados por Amaral e Cançado (2015), e em uma nova classe proposta, neste trabalho, com base em Pinker (1989) e em Cançado (2012), para a qual apresentamos evidências de não ser composta apenas de VR.

\section{Referências}

AMARAL, L.; CANÇADO, M. Argument structure of activity verbs in Brazilian Portuguese. Semantics-Syntax Interface, v. 2, n. 2, p. 115-140, 2015.

CANÇADO, M. Verbos Psicológicos: uma classe relevante gramaticalmente? Revista Veredas, v. 16, n. 2, p. 1-18, 2012.

CANÇADO, M.; AMARAL, L. Introdução à Semântica Lexical: papéis temáticos, aspecto lexical e decomposição de predicados. Petrópolis: Editora Vozes, 2016.

CANÇADO, M.; GODOY, L.; AMARAL, L. Catálogo de verbos do português brasileiro: classificação verbal segundo a decomposição de predicados. v. I - Verbos de mudança. Belo Horizonte: Editora UFMG, 2013.

CANÇADO, M.; GONÇALVES, A. Lexical Semantics: classes and alternations. In: WETZELS, L.; MENUZZI, S.; COSTA, J. The Handbook of Portuguese Linguistics. Hoboken: Willey Blackwell, 2016. p. 374-391. 
CANN, R. Formal semantics: an introduction. Cambridge: Cambridge University Press, 1993.

CHAFE, W. Directionality and paraphrase. Language, v. 47, p. 1-26, 1971.

CROFT, W. Syntactic categories and grammatical relations: the cognitive organization of information. Chicago: The University of Chicago Press, 1991.

DOWTY, D. The semantic asymmetry of 'argument alternations' (and why it matters). In: MEER, G.; MEULEN, A. Making sense: from lexeme to discourse. v. 44. Groningen: Groninger Arbeiter zur germanistischen Linguistik, 20001. p. 171-186.

DOWTY, D. Thematic proto-roles and argument selection. Language, v. 67, n. 3, p. 547619, 1991.

FILLMORE, C. J. Subjects, speakers and roles. In: DAVIDSON, D.; HARMAN, G. (ed.) Semantics of natural language. Dordrech: D. Reidel Publishing Company, 1972.

FILLMORE, C. J. The grammar of hitting and breaking. In: JACOBS, R.; ROSENBAUM, P. Reading in English Transformational Grammar. Waltham: Ginn, 1970. p. 120-133.

FILLMORE, C. J. The case for case. In: BACH, E.; HARMS, R. (ed.). Universals in linguistic theory. New York: Holt, Rinnehart and Winston, 1968. p. 1-88.

GLEITMAN, L. et al. Similar, and similar concepts. Cognition, v. 58, p. 321-376, 1996.

GODOY, L. Os verbos recíprocos no PB: interface sintaxe-semântica lexical. 2008. Dissertação (Mestrado em Estudos Linguísticos) - Faculdade de Letras, Universidade Federal de Minas Gerais, Belo Horizonte, 2008.

GRIMSHAW, J. Words and structure. Stanford: CSLI Publications/University of Chicago Press, 2005.

GRIMSHAW, J. Argument structure. Cambridge, MA: The MIT Press, 1990. 
- Análise da relevância da propriedade semântica reciprocidade nos verbos recíprocos intransitivos do Português Brasileiro

HARLEY, H. How do verbs get their names? Denominal verbs, manner incorporation and the ontology of verb roots in English. In: ERTESCHIK-SHIR, N.; RAPOPORT, T. The syntax of aspect: Deriving thematic and aspectual interpretation. Oxford: Oxford University Press, 2005. p. 42-64.

HURST, P. The Sintax of Reciprocal Constructions. In: BUTT, M.; HOLLOWAY, K. Proceedings of the LFG10 Conference. CSLI Publications, University of Melbourne, p. 311-330, 2010. Disponível em: https://goo.gl/TtHH2z. Acesso em: 04 mar. 2017.

IGNÁCIO, S. O caso comitativo. Revista CON(TEXTOS) Linguísticos, v. 3, n. 3, p. 11-25, 2009. Disponível em: https://goo.gl/3z7usT. Acesso em: 22 out. 2016.

ILARI, R. Dos problemas de imperfeita simetria. Cadernos de Estudos Linguísticos, v. 13, p. 49-65, 1987.

JACKENDOFF, R. Semantic structures. Cambridge: MIT Press, 1990.

JACKENDOFF, R. Semantics and Cognition. Cambridge: MIT Press, 1983.

JACKENDOFF, R. Semantic Interpretation in Generative Grammar. Cambridge: MIT Press, 1972.

JONES, M. Cognate objects and case-filter. Journal of Linguistics, v. 24, p. 89-110, 1988.

LEVIN, B. What is the best grain-size for defining verb classes. In: Conference on Word Classes: Nature, Typology, Computational Representations, Second TRIPLE International Conference. Rome: Università Roma Tre, 2010. p. 24-26.

LEVIN, B. Objecthood: an event structure perspective. Proceedings of CLS 35, v. 1: The Main Session, Chicago Linguistic Society, University of Chicago, Chicago, IL, p. 223-247, 1999.

LEVIN, B. English Verb Classes and Alternations: A Preliminary Investigation. Chicago: University of Chicago Press, 1993.

LEVIN, B.; RAPPAPORT HOVAV, M. Argument Realization. Cambridge: Cambridge University Press, 2005. 
PERLMUTTER, D. Impersonal passives and the Unaccusative Hypothesis. Berkeley Linguistics Society 4, p. 157-189, 1978.

PESETSKY, D. M. Events in the Semantics of English. Cambridge: MIT Press, 1995.

PINKER, S. Learnability and Cognition: The acquisition of argument structure. Cambridge: MIT Press, 1989.

RAJAGOPALAN, K. Quando 2+3 não é igual a 3+2: a semântica e a pragmática das construções simétricas em língua natural. Cadernos de Estudos Linguísticos, n. 13, p. 6796, 1987.

RAPPAPORT HOVAV, M.; LEVIN, B. Reflections on Manner/Result Complementarity. In: RAPPAPORT HOVAV, M.; DORON, E.; SICHEL, I. Syntax, lexical semantics, and event structure. Oxford: Oxford University Press, 2010. p. 21-38.

RAPPAPORT HOVAV, M.; LEVIN, B. Building Verb Meanings. In: BUTT, M.; GEUDER, W. (ed.). The Projection of Arguments: Lexical and Compositional Factors, CSLI Publications. Stanford: CA, 1998. p. 97-134.

ROSS, J. R. Act. In: DAVIDSN, D.; HARMAN, G. Semantics of natural language. Dordrecht: D. Reidel, 1972. p. 70-126.

SILONI, T. Reciprocal verbs. In: FALK, Y. (ed.). Proceedings of Israel association for theoretical linguistics, 17, 2001. Disponível em: http://atar.mscc.huji.ac.il/ english/ IATL/17/. Acesso em: 02 fev. 2017.

SILONI, T. Reciprocal verbs and symmetry. Natural Language \& Linguistic Theory, v. 30, p. 261-320, 2012. Disponível em: https://goo.gl/pbvynd. Acesso em: 02 fev. 2017.

VAN VALIN, R. Exploring the Syntax-Semantics Interface. Cambridge: Cambridge University Press, 2005.

VENDLER, Z. Linguistics in Philosophy. Ithaca: Cornell, 1967. 
- Análise da relevância da propriedade semântica reciprocidade nos verbos recíprocos intransitivos do Português Brasileiro

COMO CITAR ESTE ARTIGO: BECHIR, Thaís. Análise da relevância da propriedade semântica reciprocidade nos verbos recíprocos intransitivos do Português Brasileiro. Revista do GEL, v. 16, n. 1, p. 9-36, 2019. Disponível em: https://revistadogel.gel.org.br/

DOI: http://dx.doi.org/10.21165/gel.v16i1.1840

Submetido em: 31/05/2017 | Aceito em: 07/06/2019. 